\title{
Ticagrelor induced systemic inflammatory response syndrome
}

\author{
Philipp Krisai ${ }^{1,2^{*}}$, Manuel Haschke ${ }^{3}$, Peter T. Buser ${ }^{4}$ and Christian Mueller ${ }^{4,2}$
}

\begin{abstract}
Background: Ticagrelor is a reversible and direct-acting oral antagonist of the adenosine diphosphate receptor P2Y12. Possible adenosine-mediated effects of ticagrelor on inflammation are complex and incompletely understood. To our knowledge, ticagrelor-induced systemic inflammatory response syndrome (SIRS) has not yet been described.

Case presentation: We report the case of an 84 years old patient presenting with SIRS subsequent to initiation of ticagrelor after implantation of two drug eluting stents. A broad diagnostic work-up for alternative causes and therapeutic measures were unrevealing. Discontinuation of the agent was followed by rapid improvement in clinical and laboratory signs of SIRS.
\end{abstract}

Conclusions: After exclusion of other causes, ticagrelor needs to be considered as a possible causative agent for SIRS. Due to the widespread use of ticagrelor, clinicians should be aware of this possible adverse drug reaction.

Keywords: Ticagrelor, SIRS, Case report, Adverse drug reaction

\section{Background}

Ticagrelor, a reversible and direct-acting oral antagonist of the adenosine diphosphate receptor P2Y12, significantly reduced death as compared to clopidogrel in patients with acute coronary syndrome [1]. This led to widespread use of the agent and implementation in current guidelines [2]. Possible adenosine-mediated effects of ticagrelor on inflammation are complex and incompletely understood [3]. Due to the lower incidence of sepsis and pulmonary adverse events as well as lower mortality in patients taking ticagrelor versus clopidogrel, such effects were previously considered to be beneficial. To our knowledge, ticagrelorinduced systemic inflammatory response syndrome (SIRS) has not yet been described.

\section{Case presentation}

We present the case of an 84 years old male presenting with dyspnea (NYHA III) and fatigue, hypotension (88/ $50 \mathrm{mmHg}$ ), tachycardia (97 bpm), and fever (38.4 ${ }^{\circ}$ Celsius) to our emergency department fulfilling 2 of 4 criteria for

\footnotetext{
*Correspondence: philipp.krisai@usb.ch

'Department of Medicine, University Hospital Basel, Petersgraben 4, 4031

Basel, Switzerland

${ }^{2}$ Cardiovascular Research Institute Basel, University Hospital Basel, Basel,

Switzerland

Full list of author information is available at the end of the article
}

SIRS [4]. Clinical examination was significant for discrete bibasal pulmonary rales and a 2/6 systolic murmur, in agreement with a preexisting, moderate mitral valve insufficiency. Initial laboratory findings showed substantially elevated C-reactive protein (CRP) (84 mg/l) and serum creatinine $(159 \mu \mathrm{mol} / \mathrm{l})$.

Recent medical history was significant for ST-elevation myocardial infarction (STEMI) 15 days prior to the current presentation with successful percutaneous coronary intervention and implantation of two drug eluting stents in the proximal right coronary artery. Other relevant comorbidities included pre-existing coronary artery disease, arterial hypertension and hypercholesterolemia. His current medication included aspirin, ticagrelor, nebivolol, olmesartan, rosuvastatin and pantoprazol, with ticagrelor initiated 15 days ago. He had no history of allergies.

Empirical antibiotic treatment with ceftriaxone was initiated in the emergency department due to suspected severe sepsis after blood and urine culture sampling. Extensive infectious disease work-up including blood cultures, a respiratory panel for the comprehensive detection of respiratory disease-causing viruses and bacteria, HIV testing, and imaging studies (CT-scan of the chest, abdominal ultrasound, transthoracic and transoesophageal echocardiography) did not reveal an infectious cause of SIRS. 
Symptoms (dyspnea and fatigue), signs (fever), and laboratory signs (CRP) of SIRS persisted despite 6 days of intravenous antibiotic treatment (Fig. 1). Further, DresslerSyndrome was considered as a differential diagnosis. However, lack of a pericardial rub, leukocytosis, pericardial effusion, or clinical and laboratory response to preemptive treatment with ibuprofen rendered it very unlikely.

After broad, unrevealing diagnostic work-up ticagrelor was suspected as the causative agent of persistent SIRS due to recent initiation and no other change in drug treatment. Accordingly, ticagrelor was discontinued at day seven and replaced by clopidogrel. No other drug was changed during the hospital stay. This was followed by a rapid improvement in symptoms as well as clinical and laboratory signs of SIRS. In clinical follow-up 2 weeks and 1 year after discharge the patient remained asymptomatic and well.

\section{Discussion}

To our knowledge this is the first report describing ticagrelor induced SIRS. The close temporal association between the initiation and discontinuation of ticagrelor with the onset and resolution of SIRS, as well as the lack of evidence for an alternative cause despite extensive diagnostic and therapeutic measures justify considering a causal relationship as possible. Two unlikely differential diagnoses remain. First, the patient could have suffered from sepsis due to viral infection which escaped detection despite the broad diagnostic work-up and by chance resolved exactly at the time of discontinuation of ticagrelor. Second, the patient could have suffered from a self-limiting non-infectious inflammatory disease without any additional rheumatologic symptoms and/or signs, which by chance started soon after the initiation of ticagrelor and also by chance resolved exactly at the time of discontinuation of ticagrelor.

A recent study showed discontinuation of ticagrelor in $17 \%$ of treated patients. Conventional side effects of ticagrelor included dyspnea, bleeding, dizziness, rash, itching and gastrointestinal adverse reactions. However the most frequent cause of discontinuation was the need for oral anticoagulation therapy [5]. Additionally frequent comorbidities in patients with ischemic heart disease like chronic obstructive pulmonary disease may influence discontinuation rates of ticagrelor. This is mainly due to known side effects including dyspnea and bleeding [6]. However evidence is missing to suggest a different use of ticagrelor in this patient population [7].

Interactions between platelet P2Y12 inhibitors and the immune system result in both favorable, including reduced incidence of sepsis and pulmonary adverse events, and adverse, including elevation of CRP and dyspnea, effects [1, 3]. Underlying mechanisms are not fully understood. Potential mechanisms are inhibition of leukocyte-platelet interactions with alterations in more downstream inflammatory processes, the inhibition of P2Y12 receptors on other cells, including dendritic and vascular smooth muscle cells, and an increase in

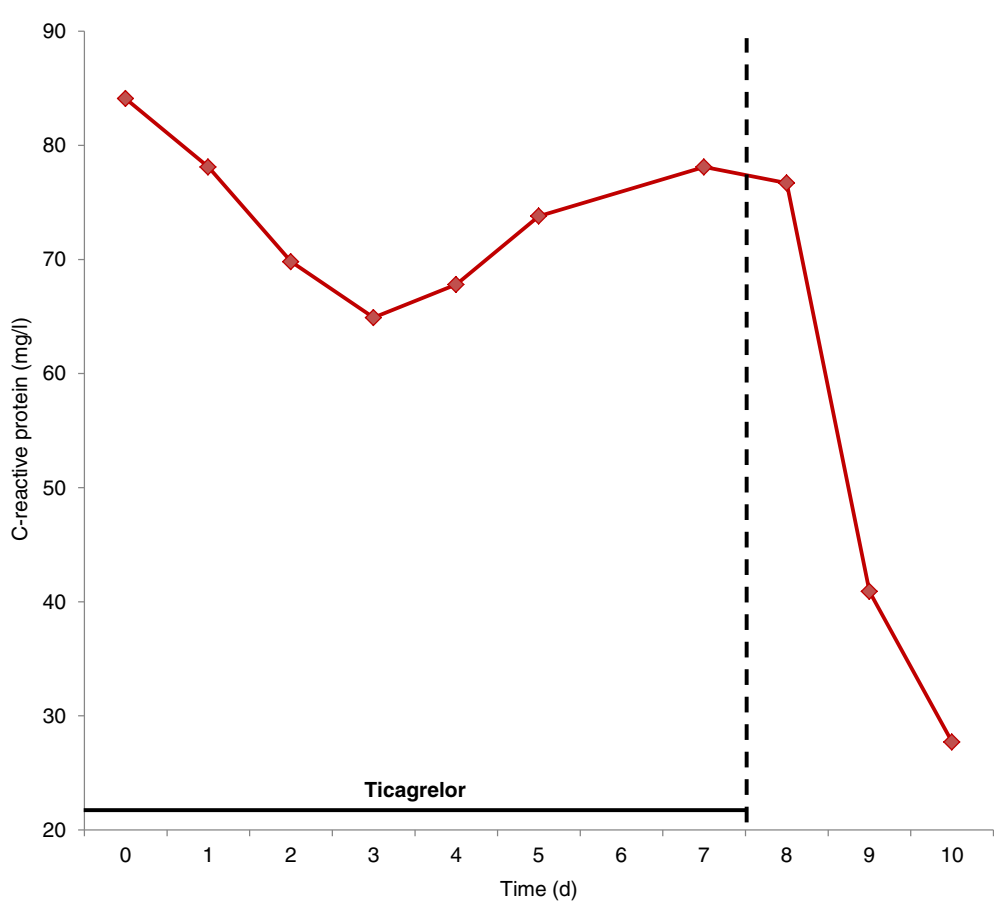

Fig. 1 C-reactive protein levels in relation to ticagrelor treatment 
extracellular adenosine [3]. Adenosine acts at low concentrations pro-inflammatory via activation of leukocyte $A_{1}$ receptors. Thereby chemotaxis of neutrophils and phagocytosis of neutrophils and macrophages are facilitated. However, at higher concentrations mostly leukocyte $\mathrm{A}_{2}$ receptors are activated with subsequent decreased release of pro-inflammatory cytokines. Ticagrelor-induced changes in adenosine levels may therefore induce an imbalance in this complex system [3].

It is therefore important to consider ticagrelor as a causative agent in a patient presenting with SIRS soon after the initiation of ticagrelor. Further research is urgently needed to better elucidate the interactions between ticagrelor and the immune system considering the widespread use of the drug.

\section{Conclusions}

Ticagrelor needs to be considered as a possible causative agent in patients presenting with SIRS whenever the diagnostic work-up for infectious causes is unrevealing.

\section{Abbreviations}

CRP: C-reactive protein; SIRS: Systemic inflammatory response syndrome

\section{Acknowledgements}

None.

\section{Funding}

No funding was received for this study.

\section{Availability of data and materials}

The data used and analysed in this manuscript is available from the corresponding author on reasonable request.

\section{Authors' contributions}

PK cared for the patient in the inpatient clinic, analyzed and interpreted the data, drafted the manuscript and made critical revision of the manuscript for important intellectual content. MH made critical revision of the manuscript for important intellectual content and analyzed the data. PB cared for the patient in the inpatient and outpatient clinic, made critical revision of the manuscript for important intellectual content and analyzed the data. CM cared for the patient in the inpatient and outpatient clinic, made critical revision of the manuscript for important intellectual content, analyzed the data and handled supervision. All authors read and approved the final manuscript.

\section{Competing interests}

The authors declare that they have no competing interests.

\section{Consent for publication}

Written informed consent was obtained from the patient for publication of this case report and any accompanying images. A copy of the written consent is available for review by the Editor-in-Chief of this journal.

\section{Ethics approval and consent to participate}

Written informed consent was obtained from the patient for participation in this case report in line with the local institutional review board of the University Hospital Basel. A copy of the written consent is available for review by the Editor-in-Chief of this journal.

\section{Author details}

'Department of Medicine, University Hospital Basel, Petersgraben 4, 4031 Basel, Switzerland. ${ }^{2}$ Cardiovascular Research Institute Basel, University Hospital Basel, Basel, Switzerland. ${ }^{3}$ Division of Clinical Pharmacology and
Toxicology, University Hospital Basel, Basel, Switzerland. ${ }^{4}$ Department of Cardiology, University Hospital Basel, Basel, Switzerland.

Received: 27 July 2016 Accepted: 14 December 2016

Published online: 06 January 2017

References

1. Wallentin L, Becker RC, Budaj A, Cannon CP, Emanuelsson H, Held C, et al. Ticagrelor versus Clopidogrel in Patients with Acute Coronary Syndromes. N Engl J Med. 2009;361:1045-57.

2. Roffi M, Patrono C, Collet J-P, Mueller C, Valgimigli M, Andreotti F, et al. 2015 ESC Guidelines for the management of acute coronary syndromes in patients presenting without persistent ST-segment elevation. Eur Heart J. 2016;37:267-315.

3. Thomas MR, Storey RF. Effect of P2Y12 inhibitors on inflammation and immunity. Thromb Haemost. 2015;114:490-7.

4. American College of Chest Physicians/Society of Critical Care Medicine Consensus Conference: definitions for sepsis and organ failure and guidelines for the use of innovative therapies in sepsis. Crit. Care Med. 1992;20:864-74

5. Biscaglia S, Campo G, Pavasini R, Tebaldi M, Tumscitz C, Ferrari R. Occurrence, causes, and outcome after switching from ticagrelor to clopidogrel in a real-life scenario: data from a prospective registry. Platelets. 2016:27:484-7.

6. Campo G, Pavasini R, Malagù M, Mascetti S, Biscaglia S, Ceconi C, et al. Chronic obstructive pulmonary disease and ischemic heart disease comorbidity: overview of mechanisms and clinical management. Cardiovasc. Drugs Ther. Spons. Int. Soc. Cardiovasc. Pharmacother. 2015;29:147-57.

7. Campo G, Pavasini R, Biscaglia S, Contoli M, Ceconi C. Overview of the pharmacological challenges facing physicians in the management of patients with concomitant cardiovascular disease and chronic obstructive pulmonary disease. Eur Heart J Cardiovasc Pharmacother. 2015;1:205-11.
Submit your next manuscript to BioMed Central and we will help you at every step:

- We accept pre-submission inquiries

- Our selector tool helps you to find the most relevant journal

- We provide round the clock customer support

- Convenient online submission

- Thorough peer review

- Inclusion in PubMed and all major indexing services

- Maximum visibility for your research

Submit your manuscript at www.biomedcentral.com/submit
) Biomed Central 